\title{
Recent Advancements in Turbo Coding with Interleave Division Multiple Access (IDMA) Technique
}

\author{
Poonam Chauhan $^{1}$, Dr. G. K. Upadhyay ${ }^{2}$ Deepti Shastri ${ }^{3}$ \\ Research Scholar, Shri Shri Venkateshwara University, Gajraula Distt: Amroha (India) ${ }^{1}$ \\ Director, Landmark Technical Campus, Didauli, Distt: Amroha (India) ${ }^{2}$ \\ Asst. Prof. (ECE), Shri Shri Venkateshwara University, Gajraula Distt: Amroha (India) ${ }^{3}$
}

\begin{abstract}
Turbo-codes have attracted a great deal of interest since their discovery in 1993. This paper reviews the reasons for this, in particular their attainment of the ultimate limits of the capacity of a communication channel. Turbo-codes promise the attainment of the 'Holy Grail' of communication theory, sought for nearly half a century to achieve the ultimate limits of capacity of a communication channel. It is not surprising, therefore, that they have very rapidly moved from the research laboratories to find practical application throughout the world and beyond it. Following their announcement in 1993, they have found a very wide range of applications, mainly in wireless communications, ranging from the third generation mobile systems to deepspace exploration. Now there is lots of advancement take place in turbo codes by the use of interleave division multiple access. The interleaver combines the output of turbo decoders. Several new interleaver designs for turbo codes are presented which illustrate the important role that the interleaver plays in these codes. The relationship between turbo codes and Low density parity check codes is explored via an explicit formulation of the parity-check matrix of a turbo code, and simulation results are given for sum product decoding of a turbo code. Our discussion encompasses analysis and optimization techniques, superposition-coded modulation, frequency domain processing schemes and the applications of IDMA in multiple-input multiple-output, relay and ad hoc environments. We demonstrate the flexibility and robustness provided by IDMA. We show that the IDMA principle can be applied to realize many potential performance gains highlighted by information theory, including coding gain, diversity gain and multi-user gain. We demonstrate that these gains can be very high, ranging from a few to tens of $d B s$, compared with conventional approaches.
\end{abstract}

Keyword: Interleavers, iterative decoding, low-density parity-check codes, convolutional codes, turbo codes

\section{Introduction}

The discovery of turbo codes [1], [2] and the subsequent rediscovery of low-density parity-check (LDPC) codes [3], [4] represent major milestones in the field of channel coding. These two classes of codes can achieve realistic bit error rates (BERs), between $10^{-6}$ and $10^{-12}$, with signal-to-noise ratios (SNRs) that are only slightly above the minimum SNR for a given channel and code rate established by Shannon's original capacity theorems. In this sense, these codes are said to be near capacity achieving codes and are sometimes considered to have solved the coding problem for the additive white Gaussian noise (AWGN) channel and its derivative channels, at least in the practical sense

LDPC codes were first discovered in 1963 [3] and shown via analysis to be a good class of binary linear block codes with a straightforward construction based on parity-check matrices with a small number of 1's pseudo randomly distributed throughout the matrix. The limited computational power of computers at that time, however, restricted computer simulations to codes with small block lengths, and their near capacity achieving[ performance was not recognized until their rediscovery. Turbo codes were discovered in 1993 and presented to the communications community with a (now famous) simulation performance curve that showed near-capacity performance at BERs down to approximately $10^{-6}$, followed by a dramatic Berror floor. turbo codes also have an interesting encoder structure consisting of the parallel concatenation of two conventional feedback shift-register-based convolutional encoders whose inputs are separated by an interleaver.

Enigmatic at first, the interleaver has subsequently been shown [5], [6] to be the essential component of turbo codes. The interleaver is the dominant factor in deter-mining the distance properties, and resulting asymptotic performance, of turbo codes. It is also the primary source of complexity, in both the theoretical and implementation senses, of the encoder. At the decoder, it is the interleaver that provides the necessary separation, or de correlation, between the two convolutional encoders and enables the low-complexity iterative turbo decoder to function effectively [8]. Indeed, the study of turbo codes has largely become the study of the design, performance, and implementation issues associated with the interleaver.

More fundamentally, turbo codes and LDPC codes are examples of codes that exhibit the random structure inherent in Shannon's original theorems, and for which there exist efficient decoding algorithms for long block lengths. Perhaps the most important commonality between turbo and LDPC codes is that they both 
utilize iterative message passing decoding algorithms, usually referred to as the turbo algorithm (TA) and the sum-product algorithm (SPA). The success of the various iterative message passing algorithms is sometimes said to have ushered in a new era of modern coding theory in which the design emphasis has shifted from optimizing some code property, such as minimum distance, to optimizing the corresponding decoder structure of the code, such as the degree profile, with respect to the behavior of a message passing decoder. The success of iterative message passing algorithms as channel decoders has also led to a new processing paradigm in which iterative algorithms are being considered in a wide variety of system components, such as channel estimation and equalization.

\section{Interleaver Design}

The design of turbo codes can be divided into two parts: design of the constituent codes, and design of the interleaver for the chosen constituent codes [5]. There are two basic methods to design the constituent codes in turbo codes. The first is based on the union bound on the BER assuming a uniform interleaver in the turbo encoder that was pioneered in [7]. The principal design rule emerging from this approach is to use recursive systematic convolutional encoders with primitive feedback polynomials in an effort to maximize the effective minimum distance $\mathrm{d}^{\text {eff }}{ }_{\text {min }}$ of the code. The second approach is based on the extrinsic information transfer (EXIT) chart introduced by ten Brink [8], [9]. Through EXIT chart analysis, ten Brink discovered that the BER performance of turbo codes at low SNRs would be greatly compromised if the memory of the constituent codes is too large or, less precisely, if the constituent codes are too be strong. There have been efforts to combine be strong and weak codes together in the turbo encoder to achieve good performance at both low and high SNRs. This includes asymmetric turbo codes [10], big-numerator little-denominator (BN-LD) turbo codes [11], and time-varying turbo codes [12]. However, symmetric turbo codes with identical constituent encoders having total encoder memories between two and four are the most common. The performance gains due to optimum selection of component code parameters are very limited for short encoder memory orders. Thus the main performance improvements in the code design can be obtained by optimizing the interleaver structure.

In this section, we consider the design of interleavers for turbo coding. The optimization of various interleaver types is discussed and their performances are compared. Interleaving is a process of rearranging the ordering of a data sequence in a one-to-one correspondence format. The inverse of this process is deinterleaving, which restores the received sequence to its original order. Interleaving has been widely used in conjunction with error control coding [13]-[15]. The classical use of an interleaver is to spread out correlated burst errors in time so that the errors within a code word appear independent. Thus, a burst channel is transformed into a random error channel at the input of the decoder and a code designed for independent error channels can be used.

In a turbo coding scheme, interleaving is employed before the information data is encoded by the second component encoder. The first role of the interleaver is to construct a long random code, because long codes can approach the Shannon capacity limit. Second, the inter-leaver breaks low-weight input sequences. A turbo code interleaver is designed to transform low-weight parity sequences of the first constituent code into high-weight parity sequences of the second constituent code, with high probability. Hence, it increases the turbo code free Hamming distance and reduces the number of lower weight codewords in the code distance spectrum. This property is referred to as the spectral thinning [6] of the distance spectrum relative to the constituent convolutional codes. The final function of the interleaver is to spread the outputs from one decoder to provide the other decoders with less correlated inputs. This improves the performance of the iterative decoding algorithm.

\section{Concept Of IDMA}

Additive white Gaussian noise (AWGN) and interference are two main hazards in communication systems. After decades of research effort, we are now quite confident in handling AWGN using forward error correction (FEC) codes, such as turbo [16] and low-density parity-check (LDPC) codes [17]. Interference still remains a problem, but progress is being made steadily [18]-[24]. Interference may come in many different forms, e.g. multiple-access interference (MAI) inter symbol interference (ISI) in multipath channels cross antenna interference (CAI) in multiple transmit antenna systems and cross layer interference (CLI) in systems involving several singling layers (such as the superposition coded modulation (SCM) scheme to be discussed below). Many techniques, such as time-division multiple-access (TDMA), frequency-division multiple-access (FDMA) and orthogonal frequency-division multiplexing (OFDM) have been developed to avoid interference during transmission. We can also treat interference as additive noise, which is the principle taken in, e.g., singleuser detection (SUD) for random waveform CDMA systems. However, these methods are mostly sub-optimal from the information theory point of view. The use of random sequences (i.e., random coding) for communication forms the core of information theory. Interestingly, interference is an inevitable consequence here, since orthogonally is not essential in an optimal communication system as envisaged by Shannon. For many years, random coding was regarded as an ingenious but only theoretical tool for the proof of channel 
capacity. The invention (and the re-invention) of pseudo-random codes such as turbo and LDPC codes [16][17], however, demonstrates the important practical impact of random coding principles. The capacity of an AWGN channel can now be approached to within a fraction of a $\mathrm{dB}$ using various pseudo-random codes. The framework of interleave-division multiple-access (IDMA) [18] is closely related to random coding. With IDMA, different users are solely distinguished by user-specific interleaves. These interleavers can be selected randomly and orthogonally is not essential. Interference among users is inevitable in IDMA but can be suppressed by a low-cost iterative multi-user detection (MUD) procedure [14]. Although IDMA was originally proposed for multiple access channels (MACs), similar principles have been studied for many other applications, e.g., broadcast systems, coded modulation, multiple antenna systems, relay and ad hoc networks. In this paper, we outline several new developments related to IDMA. In particular, we focus on the following issues:

1. The analysis and optimization of IDMA systems.

2. Superposition-coded modulation for high rate systems.

3. Single-carrier and multi-carrier IDMA systems and frequency domain processing techniques.

4. IDMA in multiple-input multiple-output (MIMO), relay and ad hoc environments.

We demonstrate the flexibility and robustness provided by IDMA. We show that the IDMA principle can be applied to realize many potential performance gains highlighted by information theory, including coding gain, diversity gain and multi-user gain. We demonstrate that these gains can be very high, ranging from a few to tens of $\mathrm{dBs}$, compared with conventional approaches.

\section{Basic IDMA Principles}

Fig. 1 shows an IDMA system over a MAC. At the transmitter for user $k$, the information sequence for user $k$ is first encoded by an FEC encoder $\left(\mathrm{ENC}_{k}\right)$ with rate $R$ and then interleaved by an interleaver $\Pi_{\mathrm{k}}$ into a chip sequence $\left\{x_{k}(j)\right\}$. A power control factor $k p$ is used before transmission, which will be discussed in the next section. Note that in Fig. 1, the conventional spreading operation in CDMA is not necessary and user separation is solely guaranteed by user-specific interleavers $\left\{\Pi_{k}\right\}$

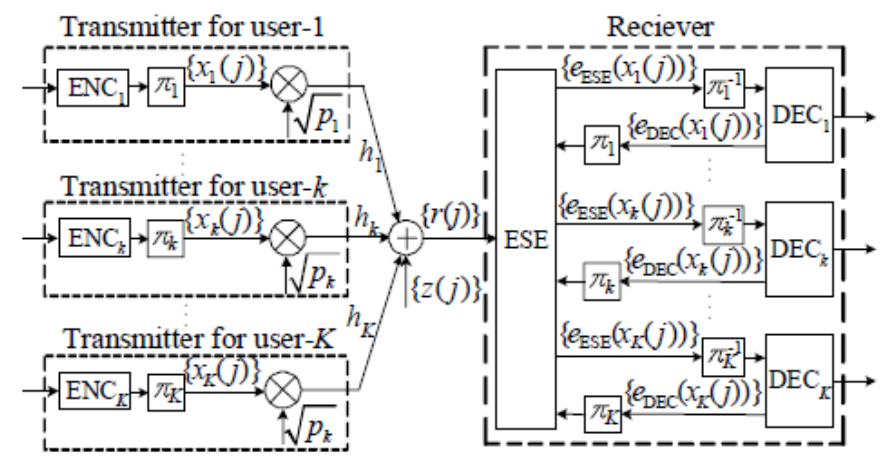

Fig: 1 The system Model of an IDMA multiple access scheme

If we consider complete synchronization at receiver and no inter symbol interference (ISI) then the received signal will be:

$r(j)=\sum_{k=1}^{K} \sqrt{P}_{k} h_{k} X_{k}(j)+Z(j)$

Where $h_{k}$ channel coefficient.

If we consider low cost detection strategy and focus on user $\mathrm{k}$ than $r(j)$ will be

$r(j)=\sum_{k=1}^{K} \sqrt{P} h_{k} X_{k}(j)+\xi_{k}(j)$

The crucial expansion is direct consequence of chip level random interleaving of IDMA.

\section{Analysis And Optimization Of IDMA}

An interesting feature of IDMA is that its performance can be quickly predicted by tracking the average SNIR of each user during the iterative process. This also leads to a fast (searching-based) design strategy for IDMA based on repeated performance evaluations 


\subsection{Fading Channels And Mug}

The advantages of IDMA become more substantial in fading channels. By the use of using a convolutional coded IDMA system with different numbers of users $K$ over a single-cell fading channel containing path loss, lognormal fading and Rayleigh fading as illustrated in Fig. 2. The system throughput is fixed at 4 bits/chip. The performance of a TDMA system with trellis coded modulation (TCM) and their corresponding channel capacities are also plotted for reference.

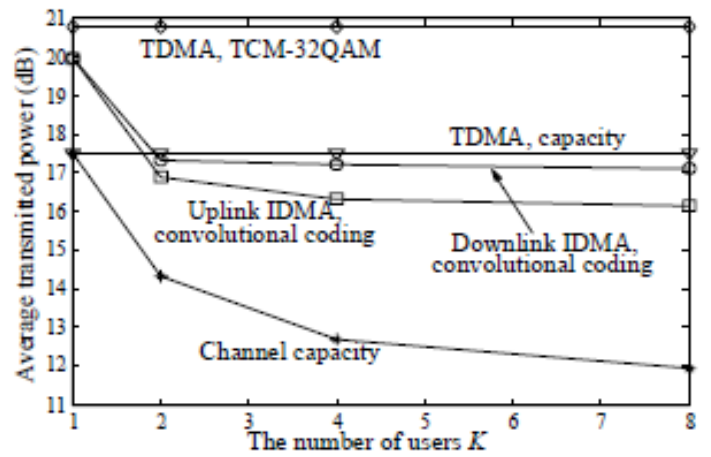

Figure 3. The average transmitted power versus the number of users $K$ for an IDMA system over a single-cell fading channel. Where outage probability is 0.01 and Desired Bit error rate is $10^{-4}$

From Fig. 2 we can see that the performance of an optimized IDMA system can be very close to the fading channel capacity (with a gap caused by the non-ideal convolutional code used) and can be improved by increasing the number of users $K$. When $K$ is large, IDMA can significantly outperform TDMA.

\subsection{Downlink Channels}

A downlink IDMA system is, in many respects, similar to the uplink system shown in Fig. 1. At the transmitter, the signals for different users are interleaved by user-specific interleavers and transmitted over a common broadcast channel. Unlike the uplink where a common MUD is shared by all users, an individual MUD is required for each user in the downlink. Therefore, the MUD cost can be a serious concern here. Nevertheless, significant multiuser gain is also achievable in the downlink, as shown in Fig. 2. This can be proved using the duality principle and has been confirmed by simulation. Since the gain is quite significant, it may justify the use of MUD in the downlink even at the cost of increased complexity, at least for a small $K$ (for which the cost increase is moderate).

\subsection{Superposition Coded Modulation}

For single-user high rate applications, we can assign all of the resources to a single user in Fig. 1. This leads to superposition-coded modulation (SCM) [19] in which several binary coded sequences, each referred to as a layer and independently encoded and interleaved, are linearly superimposed prior to transmission. A layer in SCM is roughly equivalent to a user in IDMA.SCM has several advantages over conventional coded modulation schemes such as TCM and bit-interleaved coded-modulation with iterative decoding (BICM-ID). First, with the detection method introduced above, the detection complexity of a $K$-layer SCM is linear with $K$, while that of TCM or BICM-ID with the same constellation size (2K) may grow exponentially with $K$. Thus SCM is cost effective for high throughput systems. Another advantage of SCM is its flexibility in adaptive modulation. In many scenarios including frequency selective and MIMO channels, the channel can be decomposed into a set of parallel sub-channels, of which the capacity is theoretically achieved by the waterfilling strategy. Adaptive adjustment of the coding rate in each sub-channel is needed to realize this in practical systems. With traditional coded-modulation schemes, a bank of encoders/decoders with different rates is required for this purpose, which makes the system very complicated. While with SCM, rate adaptation can be simply achieved by adjusting the number of layers. 


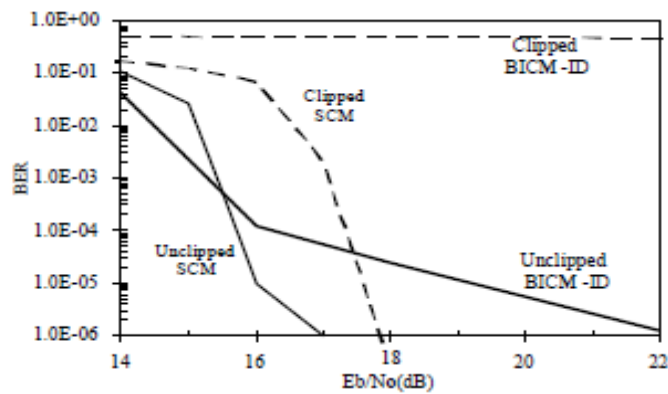

Figure 4. Performance of unclipped and clipped OFDM systems based on SCM and BICM-ID at KR $\psi=5$ bits/symbol.

A third advantage of SCM is its robustness in fast fading channels. This can be achieved by employing a relatively low-rate code for each layer to provide a high diversity order. For a fixed throughput KR, a lower single-layer rate implies more layers, which may result in increased receiver cost. Due to the superposition operation, SCM in its straightforward form has a relatively high peak-to-average power ratio (PAPR). This issue can be effectively alleviated by amplitude clipping together with soft compensation [19]. Interestingly, it turns out that SCM is advantageous with respect to PAPR when it is used in OFDM. High PAPR is an inherent problem of all OFDM systems, whether using SCM or not. However, the soft compensation method performs the best when signaling is based on SCM. For illustration, the BER performance of SCM and BICM-ID based OFDM schemes at $\mathrm{K} R \psi=5$ bits/symbol are compared in Fig. 4. It is seen that the SCM significantly outperforms BICM-ID when applied to OFDM. We are currently working on a theoretical proof for this interesting observation.

\subsection{OFDM-IDMA}

The OFDM-IDMA scheme was first introduced in [20] [21]. The basic principle of an OFDM-IDMA system is similar to that in Fig.1, except for an additional inverse FFT operation at the transmitter and an FFT operation at the receiver. These two operations transform the convolution effect of the ISI channel in the time domain into a fluctuation effect in the frequency domain (due to frequency selectiveness). The key advantage of OFDM IDMA is that MUD can be realized efficiently with complexity per user independent of the channel length and the number of users, which is significantly lower than that of other alternatives.

\subsection{Interleave-Division-Multiplexing Space-Time Coding}

If the transmitter has no CSI, the interleave-division multiplexing space-time (IDM-ST) scheme [24] shown in Fig. 7 can be employed to exploit the diversity provided by the use of multiple antennas. In Fig. 7, the information is first encoded by SCM and then segmented in a serial to parallel converter into $N$ equal-length sections to be transmitted from $N$ antennas simultaneously. The layer specific random interleaving in SCM ensures that the signal can be detected using an iterative receiver.

\section{Other Applications}

Besides cellular systems, IDMA can be applied to many other applications including those mentioned below.

\subsection{Ultra Wideband (UWB) and Sensor Systems}

IDMA is advantageous in many low-rate systems, such as UWB and sensor systems. The main advantage of IDMA in such applications is the high coding gain achievable if traditional spreading (as used in CDMA) is replaced by low-rate coding. For this purpose, the simple low-rate zigzag Hadamard code [25] can be employed. Significant coding gain can be achieved as reported in [26] for UWB systems. We expect that the same principle is applicable to sensor systems where low-power transmission is crucial to save battery life.

\subsection{Relay and Ad Hoc Networks}

An interesting concept related to IDMA is described in [27] to separate different replicas of a common signal that arrive at a destination through different relays. Suppose that the signal from a transmitter is randomly delayed before transmission and its replicas experience different delay factors through different transmission paths. If the delay difference among these paths is relatively small, then Rayleigh fading may result. However, if the delay difference is sufficiently large, these replicas may look as if they are produced using different interleavers. Using this principle, it is shown in [27] that random delay can be deliberately introduced at the relay nodes to avoid Rayleigh fading and to facilitate IDMA-type detection at the destination. This provides an efficient way to exploit the diversity provided by different transmission paths. 


\subsection{Optical Networks}

IDMA can also be applied in optical networks [28]. In the OR channel of an optical network, the linear summation operation in (1) is replaced by nonlinear OR operation, which makes it difficult to support uncoordinated multiple access. The IDMA principle provides a solution to this problem while maintaining high optical transmission rates. An IDMA architecture allowing transmission rate as high as 5.4Gbps has been proposed in [28].

\section{Conclusion}

In this paper, we have outlined the recent progress of IDMA mainly with Turbo codes as well as also with some other applications. IDMA provides many desired features for modern communications systems, in particular, robustness against interference and both high power efficiency and spectral efficiency. IDMA is also very flexible, allowing low-cost iterative detection in various channel conditions. In this paper, We have used theoretical arguments and some other practical and simulation results to explain these features of IDMA in the development and advancement of Turbo codes. Turbo codes attains lot of advancement by the use of Interleave division multiple access (IDMA).

\section{References}

[1] C. Berrou, A. Glavieux, and P. Thitimajshima, BNear Shannon limit error-correcting coding and decoding: Turbo-codes,[ in Proc. 1993 IEEE Int. Conf. Communications, pp. 1064-1070.

[2] C. Berrou and A. Glavieux, BNear optimum error correcting coding and decoding: Turbo-codes,[ IEEE Trans. Commun., vol. 44, no. 10 , pp. 1261-1271, Oct. 1996.

[3] R. G. Gallager, Low-Density Parity-Check Codes. Cambridge, MA: MIT Press, 1963

[4] D. J. C. MacKay and R. M. Neal, BNear Shannon limit performance of low density parity check codes, [ IEE Electron. Lett., vol. 32, no. 18, pp. 1645-1646, Aug. 1996.

[5] S. Benedetto and G. Montorsi, BUnveiling turbo codes: Some results on parallel concatenated coding schemes,[ IEEE Trans. Inf. Theory, vol. 42, no. 2, pp. 409-428, Mar. 1996.

[6] L. C. Pe'rez, J. Seghers, and D. J. Costello, Jr., BA distance spectrum interpretation of turbo codes,[ IEEE Trans. Inf. Theory, vol. 42, no. 6, pp. 1698-1709, Nov. 1996.

[7] S. Benedetto and G. Montorsi, BDesign of parallel concatenated convolutional codes,[IEEE Trans. Commun., vol. 44, no. 5, pp. 591-600, May 1996.

[8] S. ten Brink, BConvergence behavior of iterative decoded parallel concatenated codes, [ IEEE Trans. Commun., vol. 49, no. 10, pp. 1727-1737, Oct. 2001

[9] S. ten Brink, BDesigning iterative decoding schemes with the extrinsic information transfer chart,[ AEU Int. J. Electron. Commun., vol. 54, pp. 389-398, Nov. 2000

[10] O. Takeshita, O. Collins, P. Massey, and D. J. Costello, Jr., BA note on asymmetric turbo codes, [IEEE Commun. Letters, vol. 3, no. 3, pp. 69-71, Mar. 1999 .

[11] P.Massey, O. Takeshita, and D. J. Costello, Jr., BContradicting a myth: Good turbo codes with large memory order,[ in Proc. Int. Symp. Information Theory, 2000, p. 122.

[12] F. Jiang, M. Becker, and L. C. Pe'rez, BTime-varying turbo codes,[ IEEE Commun. Letters, vol. 8, no. 8, pp. 529-531, Aug. 2004.

[13] J. G. Proakis, Digital Communications, 3rd ed. New York: McGraw-Hill, 1995.

[14] C. B. Schlegel and L. C. Pe'rez, Trellis and Turbo Coding. New York: IEEE Press/Wiley, 2003

[15] B. Vucetic and J. Yuan, Turbo Codes Principles and Applications. Boston, MA: Kluwer, 2000.

[16] C. Berrou, A. Glavieux, and P. Thitimajshima, "Near Shannon limit error-correcting coding: turbo codes," in Proc. IEEE Int.Conf. Commun., pp. 1064-1070, Geneva, Switzerland, 1993.

[17] T. J. Richardson, M. A. Shokrollahi, and R. L. Urbanke. "Design of capacity-approaching irregular low-density paritycheck codes," IEEE Trans. Inform. Theory, no. 47, pp. 619- 637, Feb. 2001.

[18] M. Moher, "An iterative multiuser decoder for near-capacity communications," IEEE Trans. Commun., vol. 46, pp. 870-880, July, 1998 .

[19] F. Brannstrom, T. M. Aulin, and L. K. Rasmussen, "Iterative detectors for trellis-code multiple-access," IEEE Trans. Commun., vol. 50, no. 9, pp. 1478-1485, Sept. 2002.

[20] R. H. Mahadevappa and J. G. Proakis, "Mitigating multiple access interference and intersymbol interference in uncoded CDMA systems with chip-level interleaving," IEEE Trans. Wireless Commun., vol. 1, pp. 781-792, Oct. 2002.

[21] Z. Shi and C. Schlegel, "Iterative multi-user detection and error control code decoding in random CDMA," IEEE Trans. Signal Process., vol. 54, pp. 1886-1895, May 2006.

[22] J. Luo, K. R. Pattipati, P. K. Willet, and F. Hasegawa, "Near optimal multiuser detection in synchronous CDMA using probabilistic data association,” IEEE Commun. Lett., vol.5, no. 9, pp. 361-363, 2001.

[23] R. Zhang and L. Hanzo, "EXIT chart based joint code-rate and spreading-factor optimization of single-carrier interleave division multiple access," in Proc. WCNC, HongKong, March 11-15 2007.

[24] J. Ch. Fricke, M. Sandell, J. Mietzner, and P. A. Hoeher, "Impact of the Gaussian approximation on the performance of the probabilistic data association MIMO decoder,” EURASIP Journal on Wireless Commun. and Networking, vol. 2005, no. 5, pp. 796800, Dec. 2005.

[25] J. Tong, Li Ping, and X. Ma, “On superposition coding with peak-power limitation,” in Proc. IEEE Int. Conf. on Commun., ICC'06, Istanbul, Turkey, June 11-15, 2006.

[26] L. Liu, W. K. Leung, and Li Ping, "Simple chip-by-chip multiuser detection for CDMA systems," in Proc. IEEE VTC'2003Spring, Jeju, Korea, Apr. 2003, pp. 2157-2161.

[27] Q. Guo, X. Yuan, and Li Ping, "Multi-user detection techniques for Potential 3GPP long term evolution (LTE) schemes," $6^{\text {th }}$ International Workshop on Multi-Carrier Spread Spectrum (MC-SS 2007), Herrsching, Germany, May 07-09, 2007.

[28] I. Mahafeno, C. Langlais, and C. Jego, "OFDM-IDMA versus IDMA with ISI cancellation for quasi-static Rayleigh fading multipath channels," in Proc. 4th Int. Symp. on Turbo Codes \& Related Topics, Munich, Germany, Apr. 3-7, 2008. 